\title{
Targeting the metabolism of leukemia stem cells as a novel therapeutic strategy
}

\author{
Emmanuel A Ho, Neal M Davies \\ Faculty of Pharmacy, University of Manitoba, Winnipeg, Manitoba, Canada
}

Citation

Provenance

Dates

Copyright

Correspondence address

Email address

Competing interests

Abbreviations

Keywords
Ho EA, Davies NM. Targeting the metabolism of leukemia stem cells as a novel therapeutic strategy. Drugs in Context 2013; 212252. doi: $10.7573 /$ dic. 212252

Invited ; externally peer reviewed

Submitted: 9 February 2013; Accepted, subject to peer review: 18 February 2013; Published: 25 February 2013

(c) 2013 Ho EA, Davies NM. This is an open-access article distributed under the terms of the Creative Commons Attribution License (CC-BY-NC-ND 3.0) which allows unrestricted sharing, copying and distribution for personal use provided it is properly attributed. Commercial use is not permitted.

Dr Neal Davies, Dean and Professor, University of Manitoba, Faculty of Pharmacy, Winnipeg, Manitoba, Canada

Neal.Davies@ad.umanitoba.ca

Emmanuel A Ho and Neal M Davies have declared that there are no competing interests

AML, acute myeloid leukemia; CSC, cancer stem cell; LSC, leukemia stem cell; ROS, reactive oxygen species

acute myeloid leukemia; BCL-2; cancer stem cell; leukemia stem cell; reactive oxygen species
Acute myeloid leukemia (AML) is a cancer with a high mortality rate. AML affects blood cells in the bone marrow and has a fiveyear survival rate of $\approx 24 \%$ [1]. Standard treatment strategies for AML include chemotherapy, radiotherapy, and hematopoietic stem cell transplantation [2]. Due to the heterogeneous population of cancer cells and a lack of understanding of AML, therapies are often not successful.

The pathogenesis of AML is not clear and is controversial. Some authors believe that AML arises as a result of transformed hematopoietic stem cells, whereas others believe AML to be a result of genetic events occurring in mature progenitor cells [3].

For the past several decades, experimental data have shown that a subpopulation of cancer cells called cancer stem cells (CSCs) possess all the characteristics of normal stem cells (self-renewal, differentiation into various cell types) and may play a part in the development of cancer. Hence, researchers are attempting to better delineate the scientific understanding of the biology of CSCs and their role in cancer development.

Recently, researchers from the University of Rochester Medical Center (New York, NY, USA) reported that leukemia stem cells (LSCs), in comparison with non-tumorigenic cells, typically have a lower rate of energy metabolism and lower cellular

Drugs in Context - The journal of interventions in clinical practice This full text PDF downloaded from www.drugsincontext.com Drugs in Context 2013; 212252. ISSN 1740-4398

Copyright (C) 2013 Ho EA, Davies NM. Distributed under the terms of the Creative Commons Attribution License CC-BY-NC-ND 3.0

No other uses without permission. oxidative status with low levels of reactive oxygen species (termed "ROS-low"). Furthermore, it was shown that LSCs overexpress the gene BCL-2, an inhibitor of mitochondrial-initiated apoptosis that has been shown to confer chemoresistance in cancer cells, so ROS-low LSCs aberrantly overexpress BCL-2. In that study, the authors demonstrated that treatment using small molecular inhibitors of BCL-2 could impair the metabolic activity of chemotherapy-resistant LSCs, resulting in the eradication of BCL-2. Hence, reduced oxidative phosphorylation and selective eradication of quiescent LSCs is possible through BCL-2 inhibition. These results were published on 17 January 2013 in Cell Stem Cell [4]. These findings could be of appreciable importance if the sui generis physiology of ROS-low LSCs can provide an avenue to be exploited by selective drug targeting via disruption of the BCL-2-dependent oxidative phosphorylation in CSCs.

One of the major difficulties in developing effective cancer therapies is the inability to kill cancer cells without damaging normal cells. Hence, researchers have attempted to utilize the "signature" aspects of cancer as unique targets for therapy development (i.e., overexpression of certain markers, activation/ deactivation of genes). In this case, targeting the metabolism of LSCs may be relevant for the treatment of AML. However, the variability within and between patients with ROS-low CSC during disease pathogenesis must be determined with regard to clinical applicability. Furthermore, whether or not this strategy is applicable towards the treatment of other cancers remains to be elucidated.

A low oxidative state as a potentially frequent property of CSC and the lack of glycolytic activity in stem cells may be inherent features of cancers. If this hypothesis is scientifically valid, then 
therapeutic strategies based on the assumption that tumors are reliant preferentially on glycolysis may fail the null hypothesis. Moreover, the paradoxical dependence on oxidative phosphorylation of the ROS-low LSC-enriched subset of CSC is part of a capacious metabolic adaptation of these cells because they successfully maintain survival despite a dramatically reduced overall metabolic rate. Identification of BCL-2 inhibitors as LSC targeting agents (together with the apparent absence of significant toxicity to normal cells) is intriguing because it provides a basis for the investigation of therapies at disease stages where targeting residual LSCs may be essential, such as in consolidation therapy or maintenance treatment during remission.

Nonetheless, the findings described by Lagadinou et al. in 2013 [4] are provocative and a syllogistic biomedical step forward towards unraveling the understanding of CSCs and their role in the development and progression of cancer.

\section{References}

1. Cancer.Net. Leukemia - Acute Myeloid - AML. Available at: http://www.cancer.net/cancer-types/leukemia-acute-myeloid-aml/statistics/. [Last accessed 18 February 2013].

2. Roboz GJ. Current treatment of acute myeloid leukemia, Curr Opin Oncol 2012;24;711-19.

3. Passegue E, Jamieson CH, Ailles LE, Weissman IL. Normal and leukemic hematopoiesis: are leukemias a stem cell disorder or a reacquisition of stem cell characteristics?, Proc Natl Acad Sci U S A 2003;100(Suppl 1):11842-9.

4. Lagadinou ED, Sach A, Callahan K, Rossi RM, et al. BCL-2 inhibition targets oxidative phosphorylation and selectively eradicates quiescent human leukemia stem cells. Cell Stem Cell 2013; doi: 10.1016/j.stem.2012.12.013. [Epub ahead of print] 